\title{
"Valve"-Type Retainment of Flexible Ureteroscope in the Distal Ureter
}

\author{
Nariman Gadzhiev, MD, Vladislav Grigoryev, MD, Zhamshid Okhunov, MD, \\ Nobel Nguyen, MD, Aleksei Pisarev, MD, Bairamov Hikmet, MD, and Sergei Petrov, $\mathrm{PhD}^{1}$
}

\begin{abstract}
Introduction: Flexible ureteroscopy (fURS) is an endoscopic procedure for the minimally invasive treatment of ureteral and renal calculi. Due to improvements in technique and ureteroscopic instrumentation over the past two decades, complications associated with URS are infrequent. However, in the event of an unexpected device malfunction or failure, the operating surgeon must employ prompt, resolute decision-making to resolve any intraoperative complications and avoid significant injury to the kidney or ureter.

Case Presentation: The patient was a 53-year-old male with a $7 \mathrm{~mm}$ left upper pole renal stone managed by fURS and laser lithotripsy. A ureteral access sheath (UAS) was not deployed during the procedure. During fragmentation of the stone, we were unexpectedly unable to retract the ureteroscope from the ureter. Herein, we describe the procedural details leading up to the complication and the careful maneuvering required to remove the ureteroscope without damaging the ureter or the instrument.

Conclusions: We recommend use of a UAS during fURS for the treatment of ureteral and renal calculi, if not using ureter access sheath, not advancing the ureteroscope above the stone, leaving it behind the tip of the instrument, while in the ureter. Utilization of a ureter access sheath could have possibly avoided this kind of complication.
\end{abstract}

Keywords: retained ureteroscope, ureteroscopy, kidney stones, urolithiasis, stones, ureters, complications

\section{Introduction}

D UE TO RECENT ADVANCES in endoscopic technology and surgical technique, flexible ureteroscopy (fURS) has become the treatment of choice for renal calculi as much as $2 \mathrm{~cm}$, especially in patients who are poor candidates for extracorporeal shock wave lithotripsy.

Although fURS is a minimally invasive treatment modality for nephrolithiasis, it is not devoid of procedural or instrumentation complications. In the following case presentation, we describe a complication of retainment of a flexible ureteroscope in the distal ureter due to suboptimal operative technique.

\section{Presentation of Case}

The patient is a 53-year-old male with a dense (1370 HU) $7 \mathrm{~mm}$ calculus in the upper calyx of the left kidney treated with fURS (Fig. 1). The patient was not prestented, nor alpha blockers given before the procedure. Under general anesthesia, rigid inspection of the ureter was first performed, and the decision was made to proceed without placement of a ureteral access sheath (UAS) after obtaining access to the left kidney. A flexible $7.5 \mathrm{~F}$ ureteroscope (Storz Flex- $\mathrm{X}^{2}$, Storz, Inc., Germany) was introduced into the kidney over a guidewire without any difficulty. Lithotripsy using a $275-\mu \mathrm{m}$ Holmium laser fiber $(0.5 \mathrm{~J}$ and $20 \mathrm{~Hz})$ was performed to reduce the stone to small fragments for subsequent extraction. Residual stone fragments were captured with a 1.9F Tipless Nitinol Basket. Unfortunately, the residual stone did not pass through the ureteral orifice and was released from the basket. Due to reduced visibility, the ureteroscope was advanced to the proximal ureter, and the laser fiber was used to further reduce the size of the stone. During this step, the ureteroscope could not be retracted due to stone fragments impacted between the

\footnotetext{
${ }^{1}$ Department of Urology, The Federal State Institute of Public Health "The Nikiforov Russian Center of Emergency and Radiation Medicine," Saint-Petersburg, Russian Federation.

${ }^{2}$ Department of Urology, University of California, Irvine, Orange, California.

${ }^{3}$ Department of Urology, National Pirogov Medical Surgical Centre, Saint-Petersburg, Russian Federation.
}

(C) Nariman Gadzhiev et al. 2017; Published by Mary Ann Liebert, Inc. This is an Open Access article distributed under the terms of the Creative Commons Attribution License, which permits unrestricted use, distribution, and reproduction in any medium, provided the original work is properly cited. 


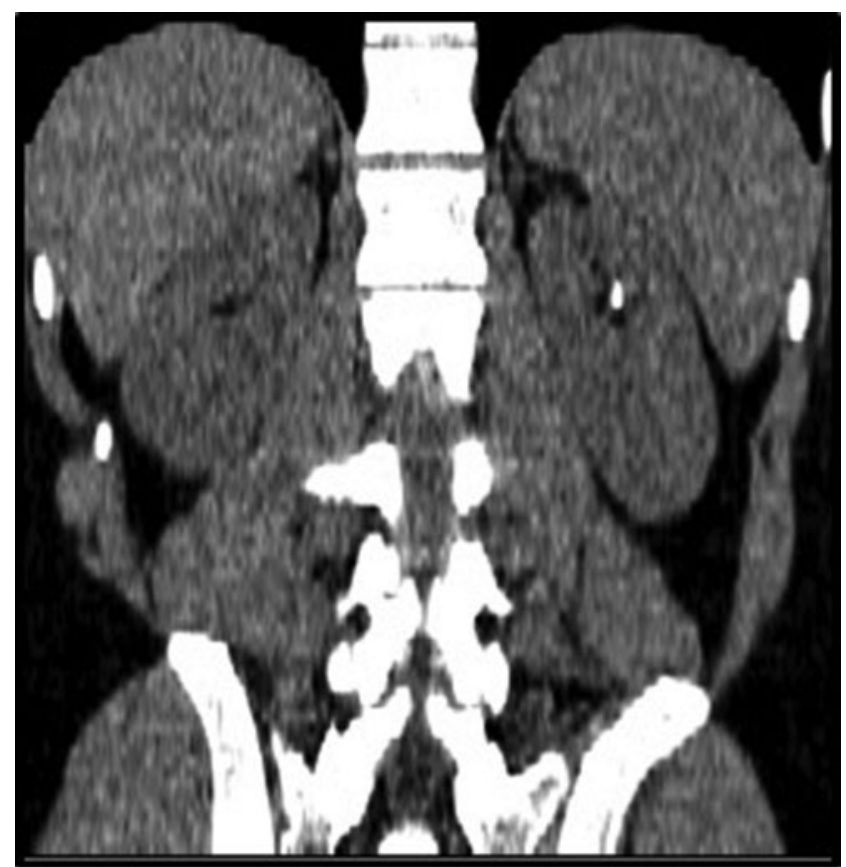

FIG. 1. CT (coronal plane). Left renal stone.

ureteroscope and the distal ureter. We were able to advance the ureteroscope toward the proximal ureter; however, the endoscope could not be withdrawn past the distal ureter. The laser fiber was subsequently removed and multiple unsuccessful attempts were made to retrieve the ureteroscope. Therefore, we proposed the following mechanism: stone fragments were abutting the coating at the distal end of the ureteroscope and the ureteral wall as shown in Figure 2. At that point, we placed a guidewire through the working channel of the ureteroscope and a Foley catheter was placed for proper drainage of the bladder. Although there was no evidence of significant bleeding, endoscopic visualization was signifi- cantly obscured and the stone was not identified in the collecting system. The patient was stable with no vital sign changes and retrograde contrast pyelography under fluoroscopy imaging showed no evidence of perforation of the collecting system.

At this point, different intervention options, including percutaneous approach to unlock the tip of the scope in case there was a locked mechanism, were considered. Open procedure was also an option in case there was evidence of perforation and significant bleeding. After lengthy discussions, we decided to proceed with the endoscopic route. Under general anesthesia, with addition of muscle relaxant, and after a period of 10 minutes to give the ureteral wall time to accommodate the instrument, attempts to remove the scope were continued in a gentle manner to avoid any damage to the ureter. Gentle rotational, back and forth maneuvers under direct visual control were attempted with moderate application of traction (Fig. 3). After a few attempts, the instrument was freed from the ureter. After the removal of the scope, which showed no evidence of malfunction, retrograde pyelography was performed with no evidence of pelvic or ureteral perforation or bleeding. Careful reinsertion of the ureteroscope into the ureter revealed that the stone fragment had got retained right behind the ureteral orifice. It was fragmented and removed with a basket. A ureteral stent and a Foley catheter were placed. No visible defects were identified on the ureteroscope. Slight attenuation of the deflecting mechanism was the only consequence of the instrument impaction. The Foley catheter was removed the same day and the ureteral stent in 14 days. During the follow-up visit after a month, neither pain nor hydronephrosis confirmed by ultrasound was present at the ipsilateral site.

\section{Discussion}

Despite the fact that safety profiles of URS are well known, there are still some complications related to the instrument itself, such as poor visibility or poor deflection, which bears no harm to the patient and can be solved by simple instrument

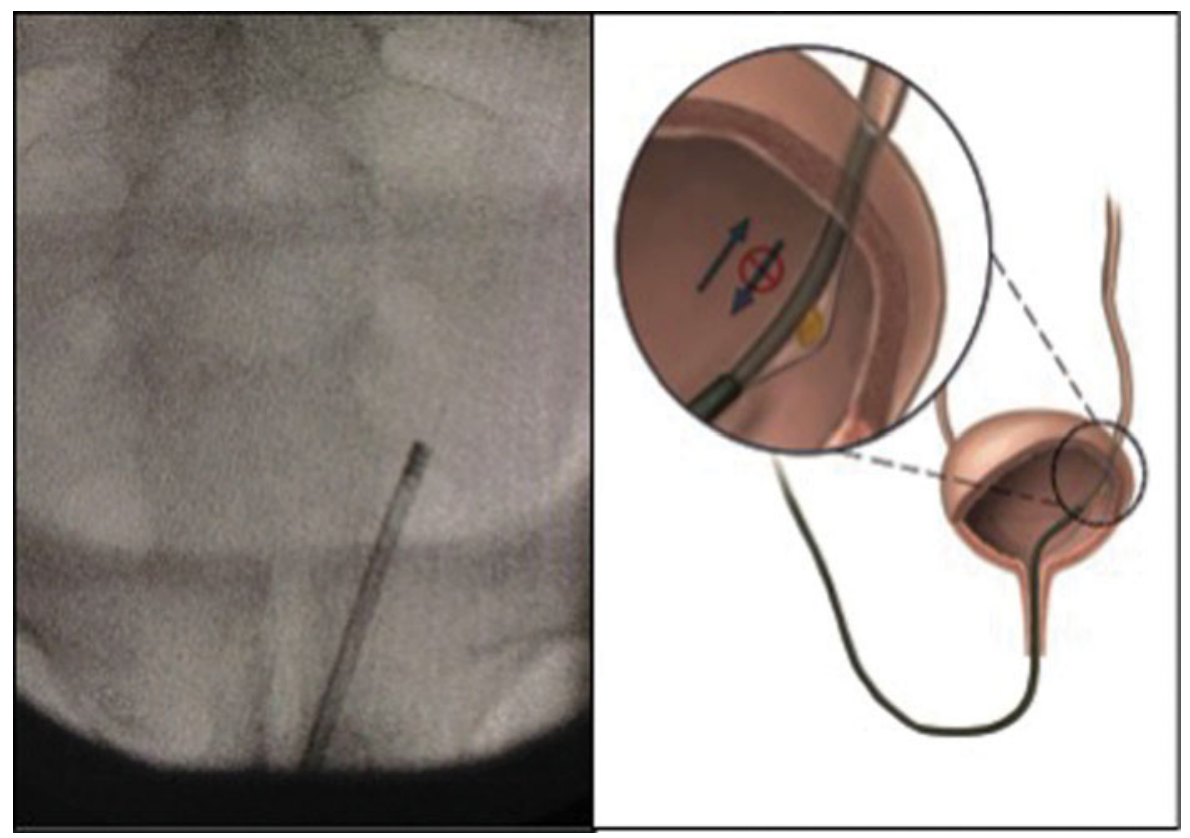

FIG. 2. X-ray film of impacted flexible ureteroscope in the distal ureter and possible mechanism of impaction. 


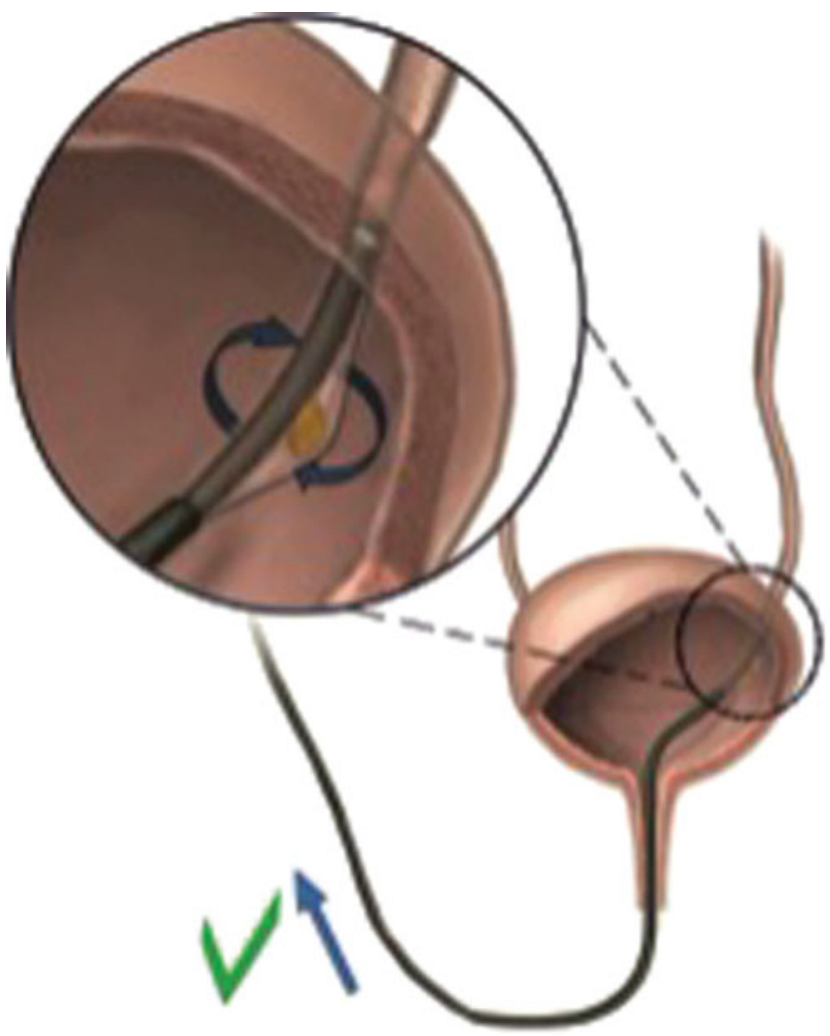

FIG. 3. Mechanism of instrument retrieval.

replacement. However, complications like locked deflection of a flexible ureteroscope while in the renal pelvis ${ }^{1}$ or retainment of the flexible ureteroscope from accordioning of the outer scope shaft skin $^{2}$ still represent significant risks. To decrease operative time and therefore reduce costs, minimize morbidity rates, and decrease the risk of damage caused by the ureteroscope, ${ }^{3}$ URS can be complemented by UAS. Unfortunately, the usage of UAS routinely in the URS for stones less than $5 \mathrm{~mm}$ is still debatable. ${ }^{4}$

The most difficult complications are those requiring additional manipulations such as for locked deflection of a flexible ureteroscope in the kidney related to improper surgical technique and ureteral avulsion due to removing excessively large stone fragments down a relatively narrow ureter. Open incision to remove a flexible ureteroscope or ureteral reconstruction will then be needed. To our knowledge,

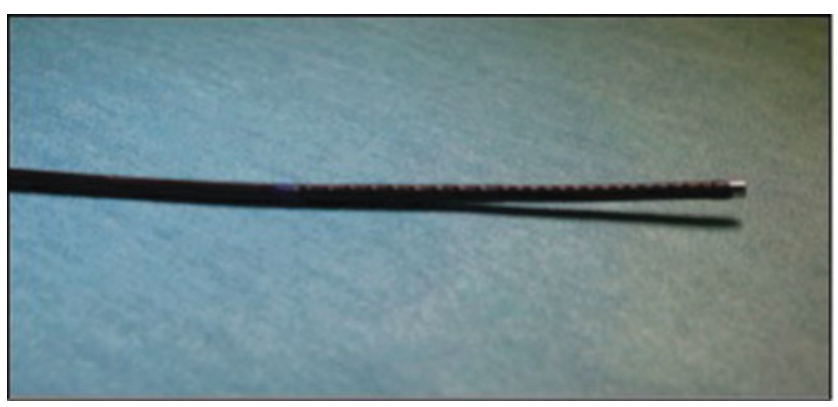

FIG. 4. Outer coating of a distal end of the flexible ureteroscope. our type of complication has never been presented before. The mechanism used was as follows: the stone fragment was abutting the outer coating of the distal end of the ureteroscope, which is fluted (Fig. 4) from one side of the ureteral wall to the other. Slow rotational movements under visual control resulted in instrument withdrawal. Also, we believe that the muscle relaxants injected during the procedure facilitated ureteral muscle relaxation and significantly helped to remove the scope.

\section{Conclusions}

The case presented herein demonstrates a novel valve-type complication due to the inability to retrieve the ureteroscope during laser lithotripsy of renal calculi. Therefore, we recommend use of a UAS during fURS and maintaining the distal tip of the ureteroscope proximal to the stone at all times during fragmentation of renal stones. Utilization of a UAS can mitigate and possibly avoid such a complication.

\section{Disclosure Statement}

No competing financial interests exist.

\section{References}

1. Hubosky SG, Raval AJ, Bagley DH. Locked deflection during flexible ureteroscopy: Incidence and elucidation of the mechanism of an underreported complication. J Endourol 2015;29:1-6.

2. Huynh M, Telfer S, Pautler S, et al. Retained digital flexible ureteroscopes. J Endourol Case Rep 2017;3:24-27.

3. Kaplan AG, Lipkin ME, Scales CD, et al. Use of ureteral access sheaths in ureteroscopy. Nat Rev Urol 2015;13:135-140.

4. Miernik A, et al. Standardized flexible ureteroscopic technique to improve stone-free rates. Urology 2012;80:1198-1202.

Address correspondence to: Nariman Gadzhiev, MD Department of Urology

The Federal State Institute of Public Health "The Nikiforov Russian Center of Emergency and Radiation Medicine”, Str. Optikov 38, kl, Fl. 35 Saint-Petersburg 197342 Russian Federation

E-mail: nariman.gadjiev@gmail.com

\begin{tabular}{|c|} 
Abbreviations Used \\
$\mathrm{CT}=$ computed tomography \\
fURS $=$ flexible ureteroscopy \\
UAS $=$ ureteral access sheath \\
$\mathrm{URS}=$ ureteroscopy
\end{tabular}

Cite this article as: Gadzhiev N, Grigoryev V, Okhunov Z, Nguyen N, Pisarev A, Hikmet B, Petrov S (2017) "Valve"-type retainment of flexible ureteroscope in the distal ureter, Journal of Endourology Case Reports 3:1, 108-110, DOI: 10.1089/cren.2017.0061. 